\title{
THE ROLE OF MODELLING AND SIMULATION IN DESIGN-BUILD PROJECTS
}

\author{
Alessandra Orsoni \\ School of Business Information Management \\ Kingston University, Kingston Hill \\ Kingston upon Thames, Surrey, KT2 7LB \\ United Kingdom \\ A.Orsoni@kingston.ac.uk
}

\author{
Nikolaos V. Karadimas \\ National Technical University of Athens \\ Multimedia Technology Laboratory \\ Heroon Polytechneiou 9, \\ Zografou Campus, 15780 Athens, Greece \\ NKaradim@central.ntua.gr
}

\begin{abstract}
KEYWORDS
Construction, Design-Build Projects, Process Modelling and Simulation, Inter-dependent Production Processes.
\end{abstract}

\begin{abstract}
The paper discusses the role of modelling and simulation techniques in large scale construction projects focusing on the opportunities and on the modelling challenges that are specific to this context of application. The paper argues that a methodology based on dynamic multi-process simulation models provides powerful decision support when assessing the performance implications of changes in the design and/or in the technology, as well as the impact of alternative courses of action during project execution. Dynamic multi-process simulation provides quantitative grounds for the understanding, analysis, and discussion among the project stakeholders, and creates the conditions for a concurrent engineering approach throughout the project's lifecycle, starting from the early stages of conceptual design. A simulation-based study, based on actual data from a construction project, is presented in the paper to illustrate these benefits.
\end{abstract}

\section{INTRODUCTION}

The analysis of change in large-scale construction projects, and especially the assessment of its impact on performance, requires a great deal of shared process understanding and communication among the project stakeholders. Large-scale construction projects involve the interests of several parties, such as the owner, the general contractor, and the different specialty contractors. Each party, depending on the type of contract, has different objectives within the project. This diversification and fragmentation of tasks and goals makes it quite difficult to establish an absolute "optimum" for the project and makes it even more difficult to express it in terms of a single performance measure.

Depending on the particular project and party of perspective, one aspect of performance may become critical to the success of the project, but in general the level of success achieved in a project can only be measured across multiple dimensions of performance (O’Connor and Miller, 1994). When dealing with complex applications, the introduction of design and technological changes impacts project performance at three levels: the system, the inter-system, and the whole project level. Specifically, the system level observes the effects of change within the system of introduction. The inter-system level tracks the effects as they ripple out to systems other than the one of first introduction. The whole project level captures the impact on the performance of the overall project. Prior research, based on extensive simulated scenario testing, has demonstrated that the impact of change can be accurately tracked at all three levels, across multiple dimensions of performance (Orsoni, 2000; Orsoni, 2001a; Orsoni 2001b; Slaughter and Orsoni, 2000).

\section{ASSESSING PROJECT PERFORMANCE IN THE PRESENCE OF CHANGE}

The complexity of large construction projects, and the multiplicity of parties and objectives involved, indicate that project performance is multi-attribute (O'Connor and Miller, 1994). While a number of different measures can be considered when analyzing the effects of project changes, for the purposes of this study a selection has been made to capture the performance impact of changes at the system, at the inter-system and at the whole project level. These measures include project duration, duration-based cost, cost of utilized resources, percentage resource utilization, and an index of workers' exposure to dangerous conditions (or danger index). Specifically, the duration-based cost represents the total cost of the project, assuming that all of the resources are present on the construction site for the entire duration of their scope of activity within the project. The cost of utilized resources is the bare cost of performing all of the project activities and tasks, excluding the resources costs of delays and wait times introduced by process interdependencies (both at the system level and at the inter-system level). The percentage of resource utilization is the ratio of these 
two costs. Worker exposure to dangerous condition is measured through an index that builds upon tabulated values of occurrence of injuries during the performance of specific construction tasks (Occupational Health and Safety Administration 1992 and 1994) over the entire duration of the project. It is important to observe that the choice of performance measures targets the assessment of the impact of inter-system process dependencies. Measures such as duration and durationbased cost in fact are dynamic measures of performance, meaning that they account for the actual duration and cost of the construction processes as determined by the inter-system process dynamics. Measures of performance such as cost of utilized resources and workers exposure to dangerous conditions, are not dynamic: they are direct functions of the bare number of man hours required to complete the project and do not account for the resource idle time caused by the inter-system process dynamics. Any discrepancies in the simulated results for these two sets of performance measures in this study is entirely determined by the effects of inter-system process links.

\section{CHALLENGES IN THE SPECIFICATIONS AND SCHEDULE OF CONSTRUCTION ACTIVITIES}

Construction projects involve the production/installation of multiple systems, such as structural, enclosure, plumbing, and electrical systems, in a same location at overlapping times (Hauptman and Hirji 1996). Specifically, because construction activities are highly labour intensive, multiple crews need to coordinate the timing of their efforts to fit the project schedule, subject to a combination of logical, technical, regulatory and resources constraints. The scheduling of construction activities is a rather challenging task: it has to be drafted ahead of time as most of the jobs are outsourced to specialty contractors. The problem with early scheduling of resources is accuracy as too many factors may affect the evolution of a project from the onset (Hendrickson and Au, 1989), typical examples are weather related delays that may cause major changes in productivity and thus completely disrupt a well-thought schedule. Another example is administrative in nature, as often special permits are required from the local government and/or from local service suppliers (electricity, water) that may delay the start either of the entire project or of the installation of a particular system. Other issues may be technical in nature, for instance may be related to the quality of related jobs for instance delays or poor quality of the materials delivered to the site, or re-work needed on some system due to lack of quality (i.e. the installation does not meet the regulatory standards or the design specifications, typical for instance of cast-in-place concrete structures). Because specialty contractors are typically involved in different projects at the same time, it is rather difficult to adjust the scheduling of their resources during the course of the project.

The remaining sections of the paper will propose and demonstrate the use of a dynamic modelling and simulation approach to support both feasibility studies (during the design phase) as well as project monitoring and control during construction. The former use of modeling and simulation aims at assessing planned changes in design and/or technology, the latter aims at coping with late changes and/or disruptions in the project schedule. Examples of both uses will be presented as part of the case study.

\section{MODELLING AND SIMULATION OF CONSTRUCTION ACTIVITIES}

Constructed facilities come in different designs and shapes to suit different aesthetic requirements and uses, however, at the microlevel (i.e. at the task-component level) their construction processes are system and material specific, so the types of activities and their requirements depend only on the system (e.g. structural system) and on the material (e.g. steel). Therefore for each system and material it is possible to specify a logical sequence of activities at the microlevel, to produce modular units as building block of more complex simulation models, where the customization of modular units is based on the actual bill of materials and on the design specifications of a particular project. Most importantly, earlier studies (Orsoni, 2000; Orsoni, 2001a; Orsoni 2001b; Slaughter and Orsoni, 2000) have shown that it is at this level of detail (taskcomponent) that intersystem process dependencies build their effects on project performance.

These considerations led to the specification and development of a library of system and material specific modules that can be dragged and dropped to suit the modeling purposes of different construction projects (Settlemeyer 2000, Murfee 1999, Pullen 1998, Murray 1998, Attai 1997, Carr 1996, Eraso 1995). A control module, or meta-model contains the specifications for the technical, logical, and regulatory constrains that dynamically determine the relative construction/installation rates among the systems. The meta-model tracks the degree of completion of each process by spatial unit of progress and regulates the activities of different crews accordingly. To implement the different modules a well-established commercial simulation package was chosen, that is Simproces ${ }^{\mathrm{TM}}$, for its ability to model shared and re-assignable resources, for the possibility to specify alternative subprocesses within each defined process, for its ability to route different components through different processing paths, and for its ability to modify the attributes of entities (e.g. materials and component) as 
they are being processed (for instance the result of a cutting process is a change in the attribute length).

Each module as developed for this library can be run as a stand alone application to test a particular system as separate from the facility, or in combination with others to model entire projects (Settlemeyer 2000, Murfee 1999, Pullen 1998, Murray 1998, Attai 1997, Carr 1996, Eraso 1995). By tracking the progress of installation for each system the meta-model ensures the synchronization of the simulated processes across modules and ensures that logical, technical, regulatory, and resources constraints are effectively respected. The system and material specific modules are design and layout independent, therefore a proper set of input data including the number, the size, and the location of each component need to be specified by the user. The input data files can be automatically generated out of a preformatted bill of materials for the project. The information about part/component location within the facility enables their aggregation by zone to keep track of the progress of installation during simulation. This information updates the state variables controlling the progress at the inter-system level, and it is a key role of the meta-model to share this information among the relevant modules.

The prototype version of the simulation model was built into a dynamic simulation environment and intended to simultaneously run the individual modules on a single CPU. However, the design of the system allows for immediate re-use of the modules in a distributed context. The meta-model in fact performs the synchronization tasks similar to those that the Run Time Infrastructure (RTI) performs in distributed applications of distributed simulation. Each system and material specific module could then be developed and more easily maintained at the sub-contractor's site as Simprocess is Java-based and thus inherently compliant with the leading distributed computing paradigms.

The models developed for this research were actually licensed to a start-up company (MOCA Systems Inc., Newton MA) that is now providing consulting services to members of the construction industry. For this purpose, an improved version of the model was implemented on a web-based platform, and made fully accessible to construction clients who can customize the modules to represent actual construction projects and use the tool as a reference for testing and improving their facility designs and their construction methods.

Simulated scenario testing based on data from an actual construction project enabled the analogical validation of the model and led to some interesting results concerning the impact of inter-system process dependencies on project performance. A first validation of the model was based on a large development project for a complex of constructed facilities including a hotel, a research facility and a parking garage. Progress and completion rates were observed and recorded for the different systems of the three facilities over a period of several months and constituted the set of reference data for the analogical validation of the model. The model was found capable of reproducing project duration and key completion milestones to a degree of accuracy of \pm 2 days for each of the facilities and their systems.

\section{CASE STUDY}

To illustrate the modeling concepts proposed in the previous section, and demonstrate the benefits of the tool, a case study is presented based on an actual construction project. The project consists on the renovation of a large residential facility, namely a student residence, based in the United States (Cambridge, MA). The project involved the replacement and the update of the mechanical and electrical systems for the facility and was subject to tight completion deadlines on each of the systems, due to the fact that work could only be carried out during a Summer period when the facility was not occupied by the students.

Two scenarios are analysed representing two different types of project changes: the first one considers a planned change in the construction methods, moving from the use of prefabricated plumbing units to their on-site fabrication, the second one considers a disruption in the project schedule due to reduced resources availability. In both cases the performance outcomes of the changes are assessed relative to a baseline configuration reflecting the original facility design and construction methods.

\section{Scenario 1: Planned Changes in the Construction Methods}

Off-site fabrication of components is often adopted to reduce installation times and reduce the interference of the process with the installation of other systems. While the original project specification (corresponding to the baseline design) involved $100 \%$ off-site fabrication of the pipe segments for the three water distribution systems (e.g. plumbing, heating and fire protection), simulated scenario testing is used in this part of the case study to decide the extent to which this decision is efficient (i.e. can actually save construction time for the project) and the extent to which the benefits of prefabrication are actually absorbed by other ongoing activities at the site, which may cause the plumbing crews to remain idle. The alternative options involved different extents of on-site fabrication, and three scenarios were customized to model the on-site fabrication for one system at a time. Specifically, the first scenario considers the on-site fabrication of fire protection pipe segments and maintains the baseline off-site fabrication of components for the heating and 
plumbing systems. Similarly, the second and third scenarios consider the on-site fabrication of components for the heating and for the plumbing systems, respectively, while maintaining the baseline off-site fabrication of components for the remaining systems.

For the first scenario, no change is observed in overall project duration because project completion time is still driven by the installation of the electrical system, as in the baseline configuration, which is not affected by the alternative considered. A significant difference can be observed between the increase in duration-based $(+0.8 \%)$ cost and the increase in cost of utilized resources $(+4 \%)$. The difference shows that while the total number of man-hours required to complete the installation of the fire protection system increases significantly in this scenario, not all of these additional man-hours translate directly into additional project duration. Some of the preparation activities can actually be performed in parallel to the installation of other units and mostly make use of resources that would otherwise be idle (baseline scenario.) This observation is supported by the fact that the percentage of resource utilization increases with respect to the baseline scenario $(+0.5 \%)$.

For the analysis of the second scenario, relevant to the on-site fabrication of components for the heating system, it is important to keep in mind that the heating system includes a supply and a return line, which run parallel to one another, whereas the fire protection system consists only of one supply line. In this scenario, by separating the supply and the return lines, no longer bundled together in a single prefabricated unit, the number of pipe segments to be installed doubles. This increase in the number of units to be placed is accounted for at the input files level. The impact of onsite fabrication at the whole project level is significantly higher than the one observed in the fire protection system.

The project completion time is still driven by the installation of the electrical system, but due to the intersystem process link between the heating and the electrical system, an increase in overall project duration can be observed $(+11 \%)$. The increase in number of man-hours required to complete the project (measured by the cost of utilized resources: $+5 \%$ ) is in percentage smaller than the increase in actual number of hours required to complete the project (measured by the duration-based cost: $+8 \%$.) This difference reveals the presence of inter-system process constraints that stretch the actual duration of the project beyond the minimum required, by increasing resource wait and idle time. This effect of on-site fabrication contrasts the one observed in the fire protection system, even though the type and level of on-site fabrication introduced in the two systems are exactly the same. An interesting contrast that leads the percentage utilization of resources to decrease, by $3 \%$, rather than increase, by $16 \%$, as observed in the previous scenario. The danger index for the whole project only increases by $1 \%$, since pipe preparation activities are performed on the ground and do not require the use of particularly dangerous equipment.

The output for the third scenario, testing the on-site fabrication of plumbing components, shows by far the highest impact on performance. In the baseline design, prefabricated bundles of pipe segments would include hot water supply and return lines, cold water supply, waste water return, drain and ventilation lines. These prefabricated segments can be installed as a single pipe and already include the required connecting elements for each of the pipes in the bundle. When comparing the installation of prefabricated bundles to the installation of each pipe as separate, the difference in the rate of installation for the two situations can be expected to be very significant. A first impact can be observed in the project duration which increases by $22 \%$. The installation of the plumbing system becomes the longest and ultimately drives the duration of the whole project. All the other performance measures increase accordingly. Table 1 presents the comparative results for the whole project.

Table 1: Performance Impact of On-Site Fabrication

\begin{tabular}{|l|c|}
\hline Performance Measure & \% Change \\
\hline Duration & $+22 \%$ \\
\hline Duration-Based Cost & $+9 \%$ \\
\hline Cost of Utilised Resources & $+12.5 \%$ \\
\hline \% Resources Utilisation & $+4 \%$ \\
\hline Danger Index & $+14.6 \%$ \\
\hline
\end{tabular}

\section{Scenario2: Manpower Shortage during Project Execution}

Because of tight completion deadlines, in the original project plan an unusually large number of resources per trade were allocated to the installation of each system, on each facility floor. Each floor was split into quadrants and two workers per trade were assigned to each quadrant. A total of 48 workers per trade were scheduled to be present on the site at all times. Under such conditions, the possibility of resources being a constraint on the project was minimal. The original project plan represents a scenario with minimal resource constraints. A new scenario was then customized to evaluate the impact of resource constraints on project performance. Specifically, the size of each crew was reduced by $50 \%$. Process performance was tested under the new conditions and compared to that of the original resource allocation plan. The new scenario represents a form of sensitivity test, to examine where and how the shortage of resources could create bottlenecks in the project. As a 
matter of fact, a shortage of manpower was experienced during project execution and the planned number of resources was never available on the site. On average the number of resources available for each crew was $50 \%$ or less than planned at all times. Significant overtime work was necessary to make up for manpower shortage and maintain the project on schedule. This scenario is then representative of the actuality project and provides an example of the type of on-line testing that project managers may want to perform when confronted with unexpected manpower shortage.

Reducing the number of resources by $50 \%$ at the crew level nearly doubles the overall duration of the project, from 52 days in the baseline configuration to 96 days. Interestingly, the duration-based cost remains virtually unchanged as compared to the baseline, showing that the major impact of reducing the number of resources is to stretch the duration of the project over a longer period of time. Consistently, both the cost of utilized resources and the danger index are exactly the same as those calculated for the baseline configuration, since no changes in design or construction methods were introduced in this scenario. In fact, if no changes are introduced in the design the number of parts and components to be installed remains unchanged and so do the construction methods, therefore the number and types of activities to be performed are exactly the same as in the baseline scenario. Consequently, the number of man-hours required to complete the project does not change, and project duration is only a function of resource availability. The same discussion applies to each of the systems individually, since this particular scenario does not introduce additional process interdependencies. However, project duration in this case is driven by the installation of the plumbing system, rather than by the installation of the electrical systems, as found for the baseline scenario.

Table 2: Performance Impact of Manpower Shortage

\begin{tabular}{|l|c|}
\hline Performance Measure & \% Change \\
\hline Duration & $+84 \%$ \\
\hline Duration-Based Cost & $-0.5 \%$ \\
\hline Cost of Utilised Resources & No Change \\
\hline$\%$ Resources Utilisation & $+0.5 \%$ \\
\hline Danger Index & No Change \\
\hline
\end{tabular}

Table 2 summarizes the percentage performance changes determined at the whole project level by the reduced resources availability. For each of the relevant performance measures the table compares the simulation outcome obtained for the alternative scenario to the ones previously obtained for the baseline scenario.
It is important to observe that besides cumulative measures of performance by system or by project, the simulation model can create records of installation progress both in space and time. Such records can be generated in two ways: either by sampling the values of the state variables at constant time intervals or by recording the times in which completion milestones are achieved based on the units of progress that are relevant to each system (e.g. floors, rooms, bays, lengths etc.). This capability makes it possible to identify the particular times and locations in which the installation rates are particularly low, and thereby target the project phases in which resources overtime is most effective. It is in this respect that the model constitutes a valuable on-line tool for project monitoring and control: it can be used to quantitatively assess the immediate consequences of unexpected changes and provides the information required to focus intervention and reaction strategies where their marginal effectiveness is the highest.

\section{CONCLUSION}

The research presented in this paper has analysed the role of simulation in design-build projects. The approach that has been examined and proposed is modular and based on dynamic multiprocess simulation to capture inter-system process dependencies and their impact on project performance. The paper has illustrated the use and the benefits of this approach through a case study, where two scenarios were considered: a planned change in the construction methods and a disruption in the construction schedule caused by the unavailability of the designated resources. These examples highlight the multiplicity of uses for simulation techniques in large construction projects. A first use is during the conceptual design, when alternative designs are assessed in conjunction with their construction methods. A simulation-based approach is especially valuable when assessing new technologies prior to their implementation. Project owners are usually reluctant in testing new designs and technologies on site, however, by means of simulation it is possible to demonstrate both their feasibility and their benefits. A second use is during bidding when alternative outsourcing strategies need to be evaluated in the allocation of jobs to different subcontractors. Finally, simulation can be used during project execution for project monitoring and control, especially when assessing the impact of alternative courses of action in response to late project changes and/or disruptions to the original schedule. These multiple uses of simulation show the relevance of this technique to support decision-making throughout the different stages of the project's lifecycle. By means of simulation the risks and the uncertainties typically associated to large construction projects can be significantly reduced across multiple dimensions of 
performance. While this use of simulation has been devised and demonstrated in application to large construction projects, other types of complex projects could similarly benefit from it. Immediate application contexts would be in shipbuilding, aircraft production and in the realization of industrial facilities.

\section{REFERENCES}

Attai, L. (1997) "Simulation to Assess Exterior Enclosure Innovations." Masters Thesis, Massachusetts Institute of Technology.

Carr, M.N. (1996) "Simulation to Assess Cast-In-Place Concrete Construction Innovations." Masters Thesis, Massachusetts Institute of Technology.

Eraso, M. (1995) "Methodology for the Economic Assessment of Construction Innovations: Simulation of Structural Steel Erection." Masters Thesis, Lehigh University.

Hauptman, O. and Hirji, K. (1996) The influence of process concurrency on project outcomes in product development: an empirical study of cross-functional teams. IEEE Transactions on Engineering Management 43: 153-164.

Hendrickson, C. and T. Au (1989) Project Management for Construction, Prentice Hall, Englewood Cliffs, New Jersey.

Murfee, W.L. (1999) "Model of Electrical Installation Process in Building Construction".Bachelors Thesis, Masachusetts Institute of Technology.

Murray, J.W. (1998) "Simulation to Assess Plumbing and Fire Protection Innovations". Masters Thesis, Massachusetts Institute of Technology.

O’Connor, J. T. and S. J. Miller (1994) Constructability Programs: Methods for Assessment and Benchmarking, Journal of Performance of Constructed Facilities, 8(1) pp 46-65.

Occupational Health and Safety Administration (1992) "Construction Accidents - The Workers' Compensation Data Base 1985-1988." U.S. Department of Labor.

Occupational Health and Safety Administration (1994) "Construction Industry: OSHA Safety and Health Standards (29 CFR Ch XVII: Subpart R-Steel Erection)." U.S. Department of Labor.

Orsoni, A. (2000) "Dynamic Process Simulation for the Design of Complex Large-Scale Systems with Respect to the Performance of Multiple Interdependent Production Processes.”. Doctoral Thesis, Massachusetts Institute of Technology.

Orsoni, A. (2001a) "Assessment of Organizational Alternatives in Complex Large Scale Projects." Proceedings of the 2001 Summer Computer Simulation Conference (SCSC 2001), Orlando, FL, July 15-19 pp. 611-616.

Orsoni, A. (2001b) "Selecting the Right Technology: Costs and Benefits of Dynamic Multi-Process Simulation in Complex, Large-Scale Applications Proceedings of the 5th World Multiconference on Systemics Cybernetics and Informatics ( SCI 2001), Orlando, FL, July 22-25 pp. 509514.

Pullen, M. (1998) "Computer-Based simulation of Construction Ativities". Technical Report, Massachusetts Institute of Technology.
Slaughter,E.S. and Orsoni, A. (2000) "Construction Process Simulation of Multiple Interdependent Processes." Proceeedings of the ASCE Construction Congress. Orlando, FL.

Settlemeyer K. (2000) "Simulation to Assess Innovations in the Erection of Wooden Structures" Masters Thesis, Massachusetts Institute of Technology.

Tatum, C. B. (1990) Integrating Design and Construction to Improve Project Performance, Project Management Journal, 21(2), 35-42.

\section{AUTHOR BIOGRAPHIES}

ALESSANDRA ORSONI is currently a senior lecturer in the School of Business Information Management at Kingston University (Kingston, UK). She received both her MS in Mechanical Engineering and her ScD in Engineering Systems Design and Innovation from the Massachusetts Institute of Technology (Cambridge, MA). Prior to joining Kingston University she was a research associate in the Department of Materials Science and Metallurgy at the University of Cambridge (Cambridge, UK) and worked as an assistant professor in the Department of Production Engineering at the University of Genova (Genova, Italy). Her research interests include modelling, simulation, and AI techniques applied to the design and management of complex industrial systems.

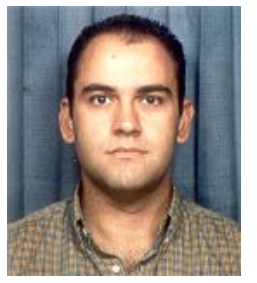

NIKOLAOS V. KARADIMAS was born in Athens, Greece and he graduated from Patra T.E.I. in 1995 with a Bachelor's degree in Electrical Engineering. He then went to receive a Bachelor's degree with Honors in Electronic Engineering and a Masters degree in Computer Science from Glasgow Caledonian University, Scotland in 1997 and 1998, respectively. He also received a Masters degree in Distributed and Multimedia Information Systems from Heriot-Watt University, Scotland in 1999. Since 2002 he is a Ph.D candidate in National Technical University of Athens. Furthermore, since 2001 he is teaching Informatics in Hellenic Army Academy and New York College, since 2003 he is teaching in T.E.I. of Chalkida and since 2005 he is teaching in Technical NCO Academy, as well. He is a member of the Greek Chamber of Engineers, member of IEEE and member of IEE. His research interests are in the fields of Databases, Optimization Techniques, Geographical Information Systems, Information Retrieval, Decision Support Systems and Multimedia. 